\title{
ÍNDICES DE CONSUMO E UTILIZAÇÃO DE CULTIVARES DE AMOREIRA POR Bombyx mori L.
}

Sérgio Antonio De Bortoli ${ }^{1}$ Marco Aurélio Montagna ${ }^{1}$ José Ednilson Miranda ${ }^{1}$ Afonso Takao Murata ${ }^{3}$ Roque Takahashi ${ }^{2}$

\section{RESUMO}

Os dados acerca da quantidade de alimento consumido, digerido, assimilado, excretado, metabolizado e convertido em biomassa avaliam o efeito de diferentes alimentos sobre o desenvolvimento de insetos. Esses parâmetros são importantes para se determinar a quantidade de alimento que é consumido e como este é aproveitado, definindo assim a qualidade do alimento utilizado. O objetivo deste trabalho foi analisar o desenvolvimento larval de Bombyx mori alimentado com quatro cultivares de amoreira, utilizando-se o método gravimétrico. $O$ trabalho foi conduzido no Laboratório de Biologia e Criação de Insetos do Departamento de Fitossanidade da Faculdade de Ciências Agrárias e Veterinárias UNESP, Jaboticabal - SP. Os cultivares estudados foram: Fernão Dias, FM Shima-Miura, Formosa e Yamada. Para a determinação do consumo e utilização do alimento, durante o quinto ínstar, as lagartas foram alimentadas três vezes ao dia $(9: 30,13: 30$ e 18:30 h). Os parâmetros analisados foram: Taxa de Consumo Relativo, Taxa Metabólica Relativa, Taxa de Crescimento Relativo, Eficiência de Conversão do Alimento Ingerido, e Eficiência de Conversão do Alimento Digerido. O cultivar Fernão Dias apresentou os maiores valores de TCR e ECD. Maiores

1 Dep. de Fitossanidade, Faculdade de Ciências Agráriạs e Veterinárias - UNESP, 14870.000 - Jaboticabal - SP.

2 Dep. de Zootecnia de Não Ruminantes, FCAV-UNESP.

3 Dep. de Zoologia, Faculdade de Filosofia, Ciências e Letras de Ribeirão Preto. 
valores de TMR, ECI e DA foram verificados em lagartas alimentadas com FM Shima-Miura. Ambos os cultivares, por estas características nutricionais, podem ser utilizados na sericicultura.

Palavras-chave: bicho-da-seda, Morus alba, método gravimétrico.

\section{ABSTRACT \\ FOOD AND CONSUMPTION UTILIZATION OF MULBERRY CULTIVAR BY Bombyx mori L.}

The data about the amount of food consumed, digested, assimilated, excreted, metabolized and transformed into biomass evaluate the effect of different foods on the development of insects. Those parameters are important to determine the amount of food that is consumed and how it is used by the insects, defining the quality of the used food. The objective of this study was to analyze the larval development of Bombyx mori fed on four mulberry cultivars, through the gravimetric method. This study was carried out under laboratory conditions at the Faculdade de Ciências Agrárias e Veterinárias UNESP, Jaboticabal, SP, Brazil. The used cultivars were: Fernão Dias, FM Shima-Miura, Formosa, and Yamada. Fifth instar caterpillars were fed three times a day (9:30 AM, 1:30 PM and 6:30 PM). The nutritional indexes studied were: Relative Consumption Rate (RCR), Relative Metabolic Rate (RMR), Relative Growth Rate (RGR), Efficiency of Conversion of Ingested (ECI), Efficiency of Conversion of Digested (ECD) and Approximated Digestibility (AD). The cultivar Fernão Dias presented the largest values of RCR and ECD. Larger values of RMR, ECI and AD were verified in larvae fed on FM ShimaMiura. Both cultivars, for these nutritional characteristics, can be used in the sericulture.-Miura showed that these larvae ingested and metabolized larger amount of food.

Key words: silkworm, Morus alba, gravimetric method. 


\section{INTRODUÇÃO}

A alimentação do bicho-da-seda é o principal fator de sucesso na produção de seda, pois o desempenho da lagarta em produzir seda é influenciado pela quantidade de alimento ingerido. Deve-se levar em conta, na alimentação destes indivíduos, a relação existente entre a composição da planta alimento, o grau de digestibilidade, a utilização desses alimentos e, principalmente a quantidade de água (Machado, 1989; Evangelista, 1994).

A quantidade de alimento fornecido aos insetos é determinante do crescimento das lagartas, além de influenciar o rendimento e a qualidade dos casulos produzidos. Paolieri (1963), ao testar diferentes números de tratos diários, notou que afetaram diretamente o desenvolvimento das lagartas. Verificou que cerca de $4,1 \%$ em peso da quantidade de folhas consumidas pelas lagartas são realmente assimilados, e que cresce a quantidade de elementos nutritivos assimilados com o aumento da quantidade de tratos.

A quantidade e a qualidade dos nutrientes componentes das folhas de amoreira podem sofrer influência de vários fatores, como a variedade de amoreira, a época e as estações do ano, a parte da planta utilizada (folhas, ramos ou brotos), a idade das folhas e, sobretudo, a incorporação ou não de nutrientes à planta (Hirano et al., 1980; Okino, 1982).

Apesar da disponibilidade de híbridos altamente produtivos, no Brasil ainda se utilizam variedades comuns e de menor produção, como a Calabresa, com 9,8\% da área plantada, a Miura, com 14,6\% e a Korin, com 18,1\% (Tinoco et al., 1991).

Ueda (1982), ao estudar a utilização do alimento pelo bicho-daseda, concluiu que a eficiência de aproveitamento aumenta conforme o desenvolvimento larval, sendo de $40 \%$ para o primeiro ínstar e de $65 \%$ para o quinto. Esta situação é justificada pela exigência menor de energia para manutenção das lagartas mais jovens.

Os dados, acerca da quantidade de alimento consumido, digerido, assimilado, excretado, metabolizado e convertido em biomassa, são muito necessários a fim de analisar o efeito de vários alimentos sobre o 
desenvolvimento dos insetos. Esses parâmetros são importantes para determinar, primeiro a quantidade de alimento consumido e como é aproveitado, para depois avaliar o papel da qualidade do alimento utilizado (Scriber \& Slansky Jr., 1993).

Takahashi et al. (1989), testando o fornecimento de folhas de amoreira a lagartas de bicho-da-seda, em intervalos de 6, 4, 3 e 2,24 horas, observaram que ocorreu diferença significativa entre os períodos. Nos três ensaios desenvolvidos o trato dos insetos com intervalo de 2,24 horas proporcionou os melhores resultados.

Evangelista (1994), ao estudar índices nutricionais para lagartas de $B$. mori alimentadas com diferentes cultivares de amoreira, observou que o alimento afeta o consumo e utilização de alimentos, mas que para no $5^{\circ}$ ínstar a tendência é não haver diferenças para os índices analisados.

O presente trabalho teve por objetivo analisar, pelo método gravimétrico, o desenvolvimento larval de Bombyx mori L., medido pelos diferentes índices de consumo e utilização de alimento, em quatro cultivares de amoreira, Morus alba L. (Moraceae).

\section{MATERIAL E MÉTODOS}

O trabalho foi conduzido nas dependências do laboratório de Biologia e Criação de Insetos do Departamento de Fitossanidade e setor de Sericicultura do Departamento de Zootecnia de Não-Ruminantes, da Faculdade de Ciências Agrárias e Veterinárias - UNESP, município de Jaboticabal, SP, cujas coordenadas são: latitude $21^{\circ} 15^{\prime} 22^{\prime \prime} \mathrm{S}$, longitude $48^{\circ} 18^{\prime} 58^{\prime \prime}$ WGr e altitude $575 \mathrm{~m}$.

O experimento foi conduzido em câmara climatizada com temperatura de $25 \pm 2^{\circ} \mathrm{C}$, umidade relativa de $80 \pm 10 \%$ e fotofase de 14 horas. Os cultivares de amoreira testados foram: Fernão Dias, FM ShimaMiura, Formosa e Yamada. Os tratos culturais estavam de acordo com os sugeridos por Takahashi (1998). O tipo de poda realizada foi em cepo, e o controle de plantas daninhas e adubação conforme recomendados por Okino (1982).

Lagartas do $3^{\circ}$ ínstar do bicho-da-seda foram recebidas da Fiação 
de Sedas Bratac S.A., Duartina, SP. Ao chegarem ao laboratório, as lagartas foram separadas em quatro grupos e colocadas em bandejas de criação, forradas com papel sulfite e cobertas com papel manteiga, formando assim os bancos de lagartas. Cada grupo recebeu como alimentação folhas de um dos cultivares de amoreira a serem estudados, uma vez ao dia. Esses bancos de lagartas foram mantidos na câmara climática. A partir do terceiro dia do quarto ínstar, as lagartas foram separadas em parcelas e instaladas em bandejas de isopor de $23,5 \mathrm{x}$ $18,0 \mathrm{~cm}$, forradas com papel sulfite e cobertas com papel manteiga. para o experimento, as lagartas foram alimentadas três vezes ao dia (9:30 h, 13:30 h, 18:30 h).

Os parâmetros estudados foram: Taxa de Consumo Relativo (TCR); Taxa Metabólica Relativa (TMR); Taxa de Crescimento Relativo (TCrR); Eficiência de Conversão do Alimento Ingerido (ECI); Eficiência de Conversão do Alimento Digerido (ECD).

Para a determinação dos índices descritos, as folhas foram cortadas longitudinalmente ao meio, retirando-se a nervura principal, sendo uma das metades pesada e destinada a alimentação das lagartas, enquanto que a outra destinou-se à composição da alíquota para determinação dos pesos nas amostragens. Todo o processo de trato das lagartas e coleta das sobras de alimentos e material de excreção foi realizado diariamente no período da manhã. De todas as amostras coletadas, bem como das alíquotas e do material alimentar fornecido, foi determinado o peso fresco; os índices apresentados foram calculados com base no peso de matéria fresca. A determinação dos parâmetros estudados foi realizada pelo método gravimétrico, utilizando três medidas básicas: peso do alimento ingerido, peso de material excretado e ganho de peso pelo inseto.

O delineamento experimental utilizado foi o Inteiramente Casualizado (DIC), com quatro tratamentos e seis repetições ; cada parcela tinha 27 lagartas de bicho-da-seda. Análises de variância, regressão linear e correlação foram efetuadas através do procedimento GLM do programa SAS. 


\section{RESULTADOS E DISCUSSÃO}

A análise dos índices nutricionais revela como os organismos respondem aos diferentes alimentos e qual alimento exerce os maiores efeitos no crescimento dos insetos. As interações entre os índices ocorre de maneira complexa, em parte envolvendo compensações homeostáticas (Scriber \& Slansky Jr., 1993).

A análise de variância dos parâmetros medidos utilizados para os cálculos dos índices nutricionais (Tabela 1) revelou diferenças significativas entre tratamentos a nível de $1 \%$ de probabilidade pelo teste F.

Tabela 1. Análise de variância do alimento ingerido, material excretado, ganho de peso de lagartas e peso médio de lagartas de bichoda-seda.

\begin{tabular}{lcccccccccc}
\hline Causas & de \\
Variação & & \multicolumn{3}{c}{ Alimento ingerido } & Material excretado & $\begin{array}{c}\text { Ganho de peso de } \\
\text { lagartas }\end{array}$ & \multicolumn{2}{c}{$\begin{array}{c}\text { Peso médio de } \\
\text { lagartas }\end{array}$} \\
\cline { 2 - 11 } & G.L. & QM & F & QM & F & QM & F & QM & F \\
\hline Tratamentos & 3 & 1,9696 & $26,07^{* *}$ & 3,0738 & $2187,76^{* *}$ & 0,2152 & $247,31^{* *}$ & 0,0353 & $61,38^{* * *}$ \\
Resíduo & 16 & 0,0755 & & 0,0014 & & 0,0009 & & 0,0006 & \\
\hline CV & & & $1,54 \%$ & & $0,54 \%$ & & $0,70 \%$ & & $0,76 \%$
\end{tabular}

ns = não signficância ao nível de 5\%; * = significância ao nível de 5\%; ** = significância ao nível de $1 \%$.

O cultivar Fernão Dias foi ingerido em quantidade superior $(\mathrm{P}<0,05)$ a Formosa e Yamada, não diferindo $(\mathrm{P}>0,05)$ de FM ShimaMiura, porém o material excretado também foi alto, sendo superior $(\mathrm{P}<0,05)$ ao dos demais cultivares (Tabela 2).

O cultivar FM Shima-Miura, além ingerido em quantidade similar a Fernão Dias e superior $(\mathrm{P}<0,05)$ a Formosa e Yamada, resultou em ganho de peso e peso médio final de lagartas superiores $(\mathrm{P}<0,05)$ aos dos outros cultivares. A maior ingestão de alimento aliada a menor quantidade de material excretado por lagartas tratadas com FM Shima-Miura 
influíram no aproveitamento do alimento, causando maior ganho de peso e maior peso médio de lagartas. Lagartas tratadas com os cultivares Formosa e Yamada, apresentaram os menores valores de ingestão, excreção, ganho de peso e peso médio.

Tabela 2. Alimento ingerido, material excretado, ganho de peso e peso médio de lagartas de quinto ínstar de lagartas de Bombyx mori alimentadas com diferentes cultivares de amoreira.

\begin{tabular}{lcccc}
\hline & \multicolumn{4}{c}{ Parâmetros de consumo } \\
\cline { 2 - 5 } Cultivar & $(\mathrm{g})$ & $(\mathrm{g})$ & & \\
\cline { 2 - 5 } & Alimento ingerido & Material excretado & Ganho de peso (g) & Peso médio (g) \\
\hline Fernão Dias & $18,45 \pm 0,52 \mathrm{a}$ & $8,05 \pm 0,04 \mathrm{a}$ & $4,33 \pm 0,03 \mathrm{~b}$ & $3,18 \pm 0,04 \mathrm{~b}$ \\
FM Shima-Miura & $18,81 \pm 0,16 \mathrm{a}$ & $6,47 \pm 0,04 \mathrm{c}$ & $4,48 \pm 0,03 \mathrm{a}$ & $3,27 \pm 0,02 \mathrm{a}$ \\
Formosa & $17,14 \pm 0,10 \mathrm{c}$ & $6,32 \pm 0,04 \mathrm{~d}$ & $4,04 \pm 0,03 \mathrm{~d}$ & $3,08 \pm 0,02 \mathrm{~d}$ \\
Yamada & $17,52 \pm 0,02 \mathrm{~b}$ & $7,02 \pm 0,02 \mathrm{~b}$ & $4,08 \pm 0,03 \mathrm{c}$ & $3,13 \pm 0,0 \mathrm{I} \mathrm{c}$ \\
\hline
\end{tabular}

letras iguais na mesma coluna não diferem entre si $(P>0,05)$ pelo teste de Duncan.

Embora não haja relatos sobre comparação especifica entre os quatro cultivares, Miranda et al. (1999) verificaram que o cultivar FM ShimaMiura, dentre cinco estudados (Miura, Korin, Calabresa, IZ 56/4 e FM Shima-Miura), foi o que propiciou maior peso de lagartas. Este resultado deve estar relacionado a aspectos quanti-qualitativos de FM ShimaMiura, como produção e qualidade nutricional foliar. FM ShimaMiura é material híbrido resultante do cruzamento das variedades Shimaguwa e Miura (Takahashi, 1996). Os híbridos têm-se mostrado mais produtivos e de maior qualidade nutricional que as variedades (Fonseca et al., 1985).

O peso acumulado ao longo do $5^{\circ}$ ínstar apresentou padrão linear de incremento. A curva resultante para o cultivar FM Shima-Miura situou-se acima das referentes aos demais cultivares, comprovando maior ganho de peso obtido com ele (Figura 1). 


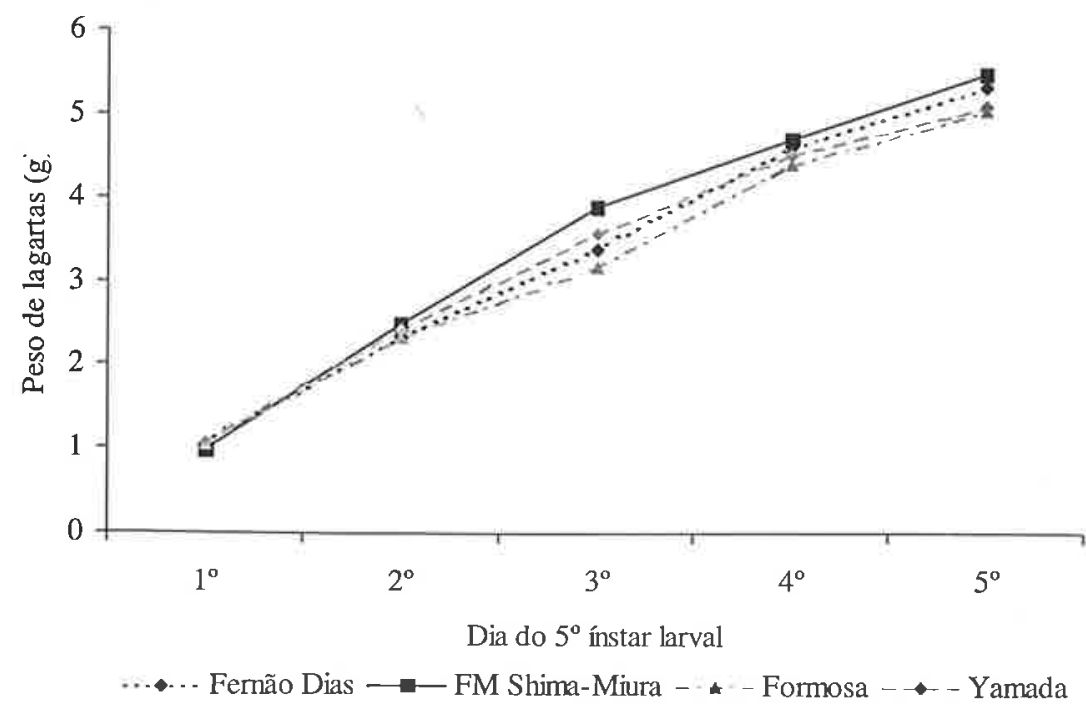

Figura 1. Peso acumulado ao longo do $5^{\circ}$ ínstar por lagartas de Bombyx mori alimentadas com diferentes cultivares de amoreira.

A análise de variância dos índices nutricionais (Tabela 3) revelou diferenças significativas entre tratamentos a nível de $5 \%$ de probabilidade para a taxa de consumo relativo (TCR) e nível de $1 \%$ de probabilidade para taxa metabólica relativa (TMR), eficiência de conversão do alimento ingerido (ECI), eficiência de conversão do alimento digerido (ECD) e digestibilidade aproximada (DA).

A taxa de consumo relativo (TCR) representa uma medida da taxa na qual o alimento entra no sistema digestivo e é provavelmente a melhor medida para a resposta comportamental ao alimento (Assal et al., 1994). Lagartas alimentadas com Fernão Dias apresentaram valores de TCR mais elevados $(\mathrm{P}<0,05)$ que aquelas que consumiram os demais cultivares, cujos valores não diferiram $(\mathrm{P}>0,05)$ entre si (Tabela 4). Um dos fatores que pode promover alterações nos valores da TCR é a quantidade de água presente no alimento. Carnaz (1992) comenta que o consumo de folhas pelas lagartas pode variar de acordo com a maior ou me- 
nor capacidade de perda de água do cultivar, vindo a influenciar diretamente outros índices de utilização do alimento.

Tabela 3. Análise de variância da taxa de crescimento relativo (TCR), taxa metabólica relativa (TMR), taxa de crescimento relativo (TCrR), eficiência de conversão do alimento ingerido (ECI), eficiência de conversão do alimento digerido (ECD) e digestibilidade aproximada (DA) de lagartas de bicho-da-seda alimentadas com diferentes cultivares de amoreira.

\begin{tabular}{|c|c|c|c|c|c|c|c|}
\hline \multirow{2}{*}{$\begin{array}{l}\text { Causas de } \\
\text { Variação }\end{array}$} & \multirow[b]{2}{*}{ GL } & \multicolumn{2}{|c|}{ TCR } & \multicolumn{2}{|c|}{ TMR } & \multicolumn{2}{|c|}{ TCrR } \\
\hline & & $\overline{Q M}$ & F & $\overline{\mathbf{Q M}}$ & $\bar{F}$ & $\overline{\mathbf{Q M}}$ & $\bar{F}$ \\
\hline Tratamentos & 3 & 0,0012 & $5,08 *$ & 0,0024 & $12,56^{* *}$ & 0,0001 & $1,90 \mathrm{~ns}$ \\
\hline Resíduo & 16 & 0,0002 & & 0,0002 & & 0,0001 & \\
\hline
\end{tabular}

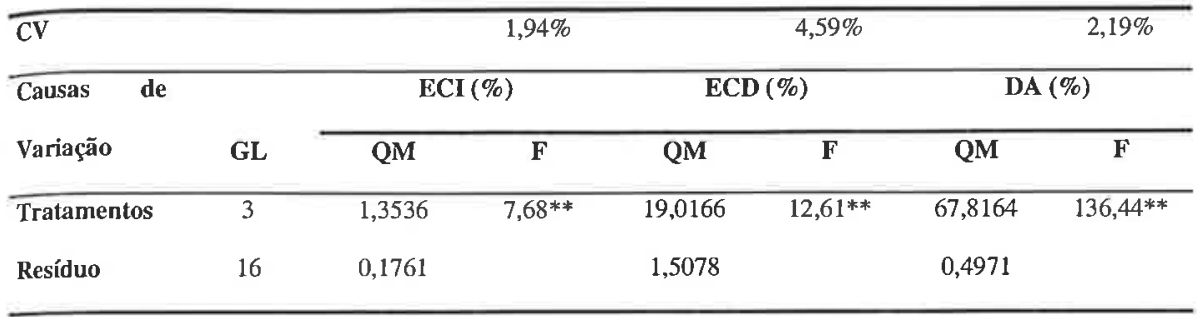

CV

$1,77 \%$

$3,16 \%$

$1,16 \%$

ns = não signficância ao nível de 5\%; * = significância ao nível de 5\%; ** = significância ao nível de $1 \%$.

A taxa metabólica relativa (TMR) representa a quantidade de alimento gasto em metabolismo por unidade de peso. É a quantidade do alimento assimilado não transformado em biomassa do inseto, mas transformado em energia (Crocomo \& Parra, 1985). As TMR de lagartas alimentadas com FM Shima-Miura e Formosa não diferiram $(\mathrm{P}>0,05)$ entre si, e foram mais elevadas $(\mathrm{P}<0,05)$ que as TMR de lagartas tratadas com Fernão Dias e Yamada, que por sua vez não diferiram $(P>0,05)$ entre si (Tabela 4). Isto significa que com FM Shima-Miura e Formosa o inseto gastou maior quantidade de alimento para manter seu metabolis- 
mo. Este dispêndio de energia pode ser compensado por um consumo também elevado, como foi verificado para FM Shima-Miura, mas não para Formosa. Independentemente do cultivar, verificou-se correlação positiva entre TMR e TCR (índice de correlação de Pearson $=0,98$; $\mathrm{P}=0,0001$ ), indicando que a taxa de consumo está relacionada com a quantidade de energia requerida para a manutenção das lagartas.

Tabela 4. Índices nutricionais de lagartas do quinto ínstar de Bombyx mori alimentadas com diferentes cultivares de amoreira.

\begin{tabular}{lcccccc}
\hline Cultivares & \multicolumn{5}{c}{ Índices nutricionais } \\
\cline { 2 - 7 } & TCR & TMR & TCrR & ECI (\%) & ECD (\%) & DA (\%) \\
\hline Fernão Dias & $0,83 \pm 0,03$ a & $0,27 \pm 0,03$ b & $0,19 \pm 0,01$ a & $23,46 \pm 0,75$ b & $41,67 \pm 2,31$ a & $56,35 \pm 1,29$ d \\
FM Shima-Miura & $0,80 \pm 0,01 \mathrm{~b}$ & $0,32 \pm 0,01$ a & $0,19 \pm 001$ a & $24,45 \pm 0,23$ a & $37,82 \pm 0,52$ b & $64,64 \pm 0,43$ a \\
Formosa & $0,80 \pm 0,01 \mathrm{~b}$ & $0,32 \pm 0,01$ a & $0,19 \pm 0,00$ a & $23,55 \pm 0,23$ b & $37,30 \pm 0,54$ b & $63,16 \pm 0,33$ b \\
Yamada & $0,80 \pm 0,00 \mathrm{~b}$ & $0,29 \pm 0,00 \mathrm{~b}$ & $0,19 \pm 0,00$ a & $23,29 \pm 0,20 \mathrm{~b}$ & $38,85 \pm 0,33$ b & $59,94 \pm 0,15 \mathrm{c}$
\end{tabular}

Letras iguais na mesma coluna não diferem entre si $(\mathrm{P}>0,05)$ pelo teste de Duncan.

A taxa de crescimento relativo (TCrR) indica o ganho de biomassa do inseto em relação ao seu peso (Miranda, 1998). Valores de TCrR de lagartas de $5^{\circ}$ ínstar de B. mori alimentadas com diferentes cultivares foram similares, indicando que o cultivar não influenciou este parâmetro (Tabela 4).

A eficiência de conversão do alimento ingerido (ECI), que mede a capacidade de o inseto utilizar para crescimento o alimento que ingere (Slansky Jr. \& Rodriguez, 1987), foi maior quando o alimento fornecido foi o cultivar FM Shima-Miura, com valor superior $(\mathrm{P}<0,05)$ ao dos demais cultivares, os quais não diferiram $(P>0,05)$ entre si (Tabela 4). Maior ECI significa que com uma mesma quantidade de alimento o inseto alcança mais cedo o peso máximo com FM Shima-Miura que quando usa outra fonte alimentar.

A eficiência de conversão do alimento digerido (ECD) representa a porcentagem de alimento assimilado convertido em substância corpo- 
ral (Miranda, 1998). A ECD foi mais elevada em lagartas tratadas com Fernão Dias, cujo valor foi superior $(\mathrm{P}<0,05)$ aos dos demais cultivares, os quais não diferiram $(\mathrm{P}>0,05)$ entre si (Tabela 4). Isto aconteceu porque a alimentação de lagartas com Fernão Dias resultou em ganho de peso relativamente alto, apesar da maior quantidade de material excretado, dentre os tratamentos (Tabela 2).

A digestibilidade aproximada (DA), também denominada de eficiência de assimilação do alimento (Slansky Jr. \& Rodriguez, 1987), representa a porcentagem do alimento ingerido efetivamente assimilado pelo inseto. FM Shima-Miura foi o cultivar que propiciou valor mais elevado de DA, superior $(\mathrm{P}<0,05)$ ao dos demais cultivares (Tabela 4). Assim, quando alimentadas com FM Shima-Miura, as lagartas digeriram mais facilmente o alimento consumido que com os outros cultivares.

Soo Hoo \& Fraenkel (1966) constataram que o crescimento está correlacionado com a digestibilidade, a eficiência de conversão e o consumo. Assim, algumas plantas conseguem influenciar positivamente $o$ crescimento ótimo quando apresentam características de boa digestibilidade, alta eficiência de conversão e alto índice de consumo. Além disso, outros fatores que podem afetar a eficiência de utilização do alimento são a quantidade de água e o teor de proteínas. Evangelista (1994), observou que a relação existente entre a composição do alimento e a quantidade de água e fator muito importantes, a ser considerado na alimentação das lagartas do bicho-da-seda. Plantas que apresentam baixa quantidade de água (30-40\% de matéria seca), tendem a apresentar menores índices de digestibilidade, quando comparados com outros com 10-20\% de matéria seca. A turgescência pode ser fator determinante para o incremento de biomassa nas lagartas, pois também influencia o rendimento e a qualidade dos casulos produzidos, conforme relatado por Paolieri (1963). Este autor cita ainda que o número de tratos diários afeta diretamente o desenvolvimento das lagartas sendo que, do total de folhas consumidas, apenas $4,1 \%$ são assimilados pelo inseto, porção que corresponde necessariamente a folhas túrgidas. Embora não tenham sido mensurada, a turgescência das folhas dos cultivares FM Shima-Miura e Formosa se mantinha por tempo mais prolongado que nas folhas de 
Fernão Dias e Yamada, podendo assim ter influenciado positivamente o desenvolvimento larval dos insetos.

\section{CONCLUSÕES}

Dentre os cultivares analisados no presente estudo, destacaramse Fernão Dias e FM Shima-Miura. O primeiro apresentou os maiores valores de TCR e ECD. Maiores valores de TMR, ECI e DA foram verificados em lagartas alimentadas com FM Shima-Miura. Estas características demonstram a boa qualidade nutricional destes materiais como alimentos do bicho-da-seda, podendo por isso serem recomendados para uso na sericicultura.

\section{REFERÊNCIAS BIBLIOGRÁFICAS}

ASSAL, O.M., BENEDETTI, R.; CAPPELLOZZA, L. \& CAPPELLOZZA, S., 1994. The Nutritional Behaviour of Three Different Races of Mulberry Silkworm (Bombyx mori) in Relation to Insegar (Fenoxycarb). Sericologia, 34:233-243.

CARNAZ, LF., 1992. Determinação de Perda de Umidade e da Produção de Seis Cultivares de Amoreira (Morus alba L.). Jaboticabal. 28p. (Trabalho de Graduação - FCAV/UNESP).

CRÓCOMO, W.B. \& PARRA, J.R.P., 1985. Consumo e Utilização de Milho, Trigo e Sorgo por Spodoptera frugiperda (J.E. Smith, 1797) (Lepidoptera: Noctuidae). Rev. Bras. Entomol., 29:225-260.

EVANGELISTA, A., 1994. Índices Nutricionais e Desempenho do Bicho-da-Seda (Bombyx mori L.) Alimentado com Diferentes Cultivares de Amoreira. Jaboticabal. 91p. (Dissertação) Mestrado - Faculdade de Ciências Agrárias e Veterinárias/UNESP.

FONSECA, A.S.; FONSECA, T.C. \& PAOLIERI, L., 1985. Caracterização de Algumas Variedades de Amoreira. Bol. Ind. Animal, 33:111-129. HIRANO, H., 1982. Variated Differences of Leaf Protein Profiles in Mulberry. Phytochem., 21:1513-1518.

MACHADO, S.A., 1989. Amoreira Substituindo a Alfafa. Cabras \& Bodes, 5:7. 
MIRANDA, J.E., 1998. Índices Nutricionais de Bombyx mori L. (Lepidoptera: Bombycidae) Alimentado com Diferentes Dietas Artificiais e Natural. Jaboticabal, 92p. (Dissertação) Mestrado - Faculdade de Ciências Agrárias e Veterinárias/UNESP.

MIRANDA, J.E.; TAKAHASHI, R. \& SILVA, A.F., 1999. Efeitos de Genótipos de Amoreira Sobre o Desenvolvimento e a Produção do Bicho-da-Seda. Rev. Agricultura, 74:169-177.

OKINO, J., 1982. Manual de Sericicultura. Bauru: Secretaria de Agricultura. 80p.

PAOLIERI, L. 1963. Quantidades de Folhas de Amoreira Consumida e de Elementos Nutritivos Assimilados pelas Larvas do Bicho-da-Seda.

Bol. Téc. Seric., 41:1-13.

SCRIBER, J.M. \& SLANSKY JR, F., 1993. The Nutritional Ecology of

Immature Insects. Ann. Rev. Entomol., 26:183-211.

SLANSKY JR., F. \& RODRIGUEZ, J.G., 1987. Nutritional Ecology of

Insects, Mites, Spiders, and Related Invertebrates. New York. J.

Wiley, 1016p.

SOO HOO, C. F. \& FRAENKEL, G., 1996. The Consumption, Digestion and Utilization of Food Plants by a Polyphagous Insect, Prodenia eridania (Cramer). J. Insect Physiol., 12:711-730.

TAKAHASHI, R., 1996. Efeito dos Diferentes Tipos de Adubação na Produção de Folhas de Amoreira (Morus alba L.), sua Influência no Desenvolvimento da Glândula Sericígena e na Produção de Casulos do Bicho-da-Seda (Bombyx mori L.). Rio Claro. 186p. (Tese) Doutorado - Instituto de Biociências/UNESP.

TAKAHASHI, R., 1998. Sericicultura. Jaboticabal: FCAV-UNESP. 135p. TAKAHASHI, R.; FELTRIN, C.A. \& GARUTA, E., 1989. Influência do

Número de Tratos Diários na Produção de Casulos do Bicho-da-Seda (Bombyx mori L.) Durante o Ano Sericícola. Ciência Zootécnica, 4:5-9. TINOCO, S.T.J.; OKAWA, H. \& ALMEIDA, R.A.C., 1991. Levantamento da Situação da Sericicultura Paulista. Boletim Técnico $n^{\circ}$ 88. Campinas: Secretaria da Agricultura. 42p.

UEDA, S., 1982. Theory of the Growth of Silkworm Larvae and its Application. JARQ, 15:180-184. 